\title{
Universal formulas for counting Nodal curves on surfaces
}

\author{
Yu-Jong Tzeng
}

\begin{abstract}
These notes are supplementary to the author's lectures at the 2010 conference on Current Developments in Mathematics, held on November 19-20 in Cambridge Massachusetts.
\end{abstract}

\section{Introduction}

The aim of enumerative geometry is to find for the number of geometric objects of specified kind, which satisfy certain incidence or tangency constraints. The history of enumerative geometry can be traced back to Italian schools, where mathematicians raised many questions about algebraic varieties in $\mathbb{P}^{N}$ and obtained important formulas. In the last twenty years, techniques from superstring theory reinvigorated enumerative geometry, resulted in many exciting results such as Candelas et al.'s formula on genus zero Gromov-Witten invariants on Calabi-Yau quintics and the YauZaslow formula on enumerating rational curves on K3 surfaces. Many classical questions were solved by introducing modern techniques. The most famous example is Kontsevich and Manin's recursive formula on $N_{d}$, the number of rational curves of degree $d$ passing through $3 d-1$ points in general position in $\mathbb{P}^{2}$.

In this article, we will report the recent progress of enumerating the number of nodal curves on algebraic surfaces.

1.1. Main results. Consider a line bundle $L$ on a complex projective smooth surface $S$. This paper attempts to answer the following question: how many reduced curves have exactly $r$ simple nodes and no higher singularities in a generic $r$-dimensional linear system of $|L|$ ? Equivalently, how many $r$-nodal curves in $|L|$ pass through $\operatorname{dim}|L|-r$ points in general position?

Göttsche [Gö] conjectured that for every $r$, the numbers of $r$-nodal curves are given by universal polynomials of four topological numbers: $L^{2}$, $L K, c_{1}(S)^{2}$ and $c_{2}(S)$ provided that the line bundle $L$ is $(5 r-1)$-very ample

(C) 2011 International Press 
(i.e. $\left.H^{0}(S, L) \rightarrow L\right|_{\xi}$ is surjective for every $\left.\xi \in S^{[5 r]}\right)$. These polynomials are universal in the sense that it only depends on $r$ and are independent of the surface and line bundle. Our first result is an algebro-geometric proof of Göttsche's conjecture.

THEOREM 1.1 (Göttsche's conjecture). For every integer $r \geq 0$, there exists a universal polynomial $T_{r}(x, y, z, t)$ of degree $r$ with the following property: given a smooth projective surface $S$ and a $(5 r-1)$-very ample (5-very ample if $r=1$ ) line bundle $L$ on $S$, a general $r$-dimensional sublinear system of $|L|$ contains exactly $T_{r}\left(L^{2}, L K, c_{1}(S)^{2}, c_{2}(S)\right)$ r-nodal curves.

Since the numbers of nodal curves for all line bundles on $\mathbb{P}^{2}$ and for primitive classes on $\mathrm{K} 3$ surfaces have been determined $([\mathbf{C H}],[\mathbf{B L}])$, all coefficients of $T_{r}$ can be computed by solving linear equations. Moreover, one can combine all universal polynomials as coefficients to define a generating function. Inspired by the Yau-Zaslow formula, Göttsche [Gö] conjectured the closed form of this generating function of $T_{r}$, which we call the GöttscheYau-Zaslow formula.

TheOREM 1.2 (The Göttsche-Yau-Zaslow formula). There exist universal power series $B_{1}(q)$ and $B_{2}(q)$ such that

$$
\begin{gathered}
\sum_{r \geq 0} T_{r}\left(L^{2}, L K, c_{1}(S)^{2}, c_{2}(S)\right)\left(D G_{2}(\tau)\right)^{r} \\
=\frac{\left(D G_{2}(\tau) / q\right)^{\chi(L)} B_{1}(q)^{K_{S}^{2}} B_{2}(q)^{L K_{S}}}{\left(\Delta(\tau) D^{2} G_{2}(\tau) / q^{2}\right)^{\chi\left(\mathcal{O}_{S}\right) / 2}}
\end{gathered}
$$

where $G_{2}$ is the second Eisenstein series $-\frac{1}{24}+\sum_{n>0}\left(\sum_{d \mid n} d\right) q^{n}, D=q \frac{d}{d q}$ and $\Delta(q)=q \prod_{k>0}\left(1-q^{k}\right)^{24}$.

If we write $q=e^{2 \pi i \tau}$ for $\tau$ on the complex upper half plane, then $G_{2}$, $D G_{2}$ and $D^{2} G_{2}$ are quasimodular forms in $\tau$ and $\Delta$ is a modular form in $\tau$. For the precise definition of quasimodular forms, see $[\mathbf{K Z}]$.

1.2. Background. On $\mathbb{P}^{2}$, the number of nodal curves is classically known as the Severi degree $N_{d, g}$, which is the number of plane curves of degree $d$ and genus $g$ passing through $3 d+g-1$ points in general position. This subject was studied by Ran ([Ran1], [Ran2]), using degeneration of $\mathbb{P}^{2}$ and an inductive procedure. In 1993, Kontsevich and Manin [KM] introduced the techniques of Gromov-Witten theory to this problem, from which they obtained a beautiful recursive formula of rational curves for all degrees. For plane curves of a higher genus, Harris and Pandharipande $[\mathbf{H P}]$ computed the Severi degrees with at most three nodes using Hilbert schemes, and Choi $[\mathbf{C h}]$ extended the result to at most four nodes using Ran's method.

The counting of nodal curves of arbitrary genus $g$ in $\mathbb{P}^{2}$ was completely solved by Caporaso and Harris $[\mathbf{C H}]$. They defined the generalized Severi 
degrees $N_{d, g}(\alpha, \beta)$ with tangential conditions and used deformation theory to derive recursive formulas of $N_{d, g}(\alpha, \beta)$. Shortly after, Vakil [Vakil] derived similar results for rational ruled surfaces.

For an arbitrary smooth projective surface, the number of nodal curves with at most three nodes can be computed directly by standard intersection theory. In 1994, Vainsencher [Va] proved the existence of universal polynomials in the case of up to six nodes. By computing the polynomials explicitly, he showed that the polynomials only depend on $L^{2}, L K, c_{1}(S)^{2}$ and $c_{2}(S)$. Later, Kleiman and Piene $([\mathbf{K P 1}],[\mathbf{K P 2}])$ refined Vainsencher's approach and generalized the result to up to eight nodes. Since their methods rely on a detailed analysis of the singularities of low codimensions, it is difficult to generalize the methods to the case of a higher number of nodes.

On algebraic K3 surfaces and primitive classes, the number of rational curves not only can be determined but also possesses a general pattern. Yau and Zaslow [YZ] discovered a surprising formula for the generating function in terms of the Dedekind function, which prompts the speculation that general modular forms may be involved. In particular, the Yau-Zaslow formula implies that the number of rational curves in an effective class $C$ only depends on the self-intersection number $C^{2}$.

The Yau-Zaslow formula was generalized by Göttsche [Gö] to arbitrary projective surface. Using Vainsencher and Kleiman-Piene's numbers, Göttsche conjectured the Göttsche-Yau-Zaslow formula, which relates the generating function to quasimodular forms. This generating function is defined by universal polynomials, which can be viewed as a virtual counting of nodal curves, especially when the line bundle is not ample enough. In addition, Göttsche observed that the generating function is completely determined by the number of nodal curves on $\mathbb{P}^{2}$ and $\mathrm{K} 3$ surfaces; thus it can be computed by the Severi degrees and quasimodular forms. A reformulation of the Göttsche-Yau-Zaslow formula gives the generating function on the number of genus $g$ curves ([Gö], remark 2.6) and this reformulation has been verified by Bryan and Leung $[\mathbf{B L}]$ for K3 surfaces and primitive ample line bundles.

The Severi degrees also have interesting properties. On $\mathbb{P}^{2}$, fixing the number of nodes and letting the degrees vary, Di Francesco and Itzykson conjectured $[\mathbf{F I}]$ that the number of plane $r$-nodal curves of degrees $d$ is a polynomial in $d$, or the node polynomial. Recently, Fomin and Mikhalkin $[\mathbf{F M}]$ proved the polynomiality with tropical geometry and found many interesting properties of node polynomials. Block $[\mathbf{B l}]$ generalized it to relative node polynomials and proved that there is a formal power series which specializes to all relative node polynomials. These results suggest that enumerating curves with broader conditions may possess a structure that has not been discovered.

We add that a symplectic proof to Göttsche's conjecture was given by A.K. Liu ([Liu1], [Liu2]), based on the work of Taubes on the equivalence of Seiberg-Witten theory and Gromov-Witten theory. Recently another proof 
was also found by Kool-Shende-Thomas [KST], using the BPS calculus and the computation of tautological integrals on Hilbert schemes by Ellingsrud, Göttsche and Lehn [EGL].

1.3. Multiplicative structure. The Göttsche-Yau-Zaslow formula expressed the generating function in terms of quasimodular forms and two unknown series $B_{1}(q)$ and $B_{2}(q)$. If the canonical divisor of a surface $S$ is numerically trivial, only the quasimodular forms appear in the generating function. Consequently, the generating function for K3 surfaces and abelian surfaces is known. The unknown series $B_{1}(q)$ and $B_{2}(q)$ can be determined using Caporaso and Harris' recursive formulas of the Severi degrees of $\mathbb{P}^{2}$. Göttsche has computed the coefficients of $B_{1}(q)$ and $B_{2}(q)$ up to degree 28 . However, we are still unable to find the closed forms for $B_{1}(q)$ and $B_{2}(q)$.

Instead of indexing on $\left(D G_{2}\right)^{r}$, we can simply use $x^{r}$ to define another generating function

$$
T(S, L)=\sum_{r \geq 0} T_{r}\left(L^{2}, L K, c_{1}(S)^{2}, c_{2}(S)\right) x^{r}
$$

Because all coefficients are universal, $T(S, L)$ is a universal power series. Moreover, $T(S, L)$ is multiplicative:

TheOREm 1.3 ([Gö], proposition 2.3). Assuming the numbers of nodal curves are given by universal polynomials, then there exist universal power series $A_{1}, A_{2}, A_{3}, A_{4}$ in $\mathbb{Q}[[x]]^{\times 1}$ such that the generating function has the form

$$
T(S, L)=A_{1}^{L^{2}} A_{2}^{L K_{S}} A_{3}^{c_{1}(S)^{2}} A_{4}^{c_{2}(S)} .
$$

While Göttsche proved this theorem by considering disjoint union of surfaces, we will give a different proof using algebraic cobordism in Section 4 . The new proof plays a central role in our approach because it demonstrates that universality is a result of algebraic cobordism structure.

The coefficients of $A_{i}$ can be determined by Caporaso-Harris $[\mathbf{C H}]$ and Vakil's [Vakil] recursive formulas on $\mathbb{P}^{2}$ and $\mathbb{P}^{1} \times \mathbb{P}^{1}$ but the closed forms are unknown. Note the generating functions in Theorems 1.2 and 1.3 can be defined for all line bundles even if they are trivial or not effective. In this setting, the $r$-th coefficient of $T(S, L)$ equals the number of $r$-nodal curves of $[S, L]$ if $L$ is sufficiently ample relative to $r$. Therefore, usually only a finite number of initial coefficients honestly represent the number of nodal curves, and after that the coefficients are virtual and hard to what they mean. This difficulty will be overcome by depicting the universal polynomials as intersection numbers $d_{r}(S, L)$ and working with $d_{r}(S, L)$ directly.

\footnotetext{
${ }^{1} \mathbb{Q}[[x]]^{\times}$is the group of units in $\mathbb{Q}[[x]]$ and the group action is defined by multiplication of power series.
} 
1.4. Approach. In this article we prove Theorem 1.3 first, and derive other theorems from this theorem. The main ingredients in our proof consist of the algebraic cobordism group $\omega_{2,1}$, the enumerative number $d_{r}(S, L)$ and the moduli stack of families of ideal sheaves. These techniques are developed in order to study the degeneration of line bundles on surfaces, and to derive a degeneration formula for the generating functions. As a consequence, we will prove when $L$ is $(5 r-1)$-very ample, the number of nodal curves in $|L|$ on $S$ only depend on the class of $[S, L]$ in $\omega_{2,1}$, which can be computed by $L^{2}, L K, c_{1}(S)^{2}$ and $c_{2}(S)$.

The algebraic cobordism theory has been developed by Levine and Pandharipande $[\mathbf{L P}]$. They call

$$
\left[X_{0}\right]-\left[X_{1}\right]-\left[X_{2}\right]+\left[X_{3}\right]
$$

a double point relation if there exists a flat family of projective schemes $\pi: \mathcal{X} \rightarrow \mathbb{P}^{1}$ satisfying the following properties: firstly, $\mathcal{X}$ is smooth and $X_{0}$ is the fiber over $0 \in \mathbb{P}^{1}$ and is smooth. Secondly, the fiber over $\infty \in \mathbb{P}^{1}$ is the union of two smooth components $X_{1}$ and $X_{2}$ intersecting transversally along a smooth divisor $D$. Thirdly, $X_{3}=\mathbb{P}\left(1_{D} \oplus N_{X_{1} / D}\right)$ is a $\mathbb{P}^{1}$ bundle over $D$. Define the addition of two schemes to be the disjoint union and the multiplication to be the Cartesian product. The algebraic cobordism ring is defined to be the ring generated by all smooth projective schemes modulo the double point relation.

Since the problem of counting nodal curves is about a surface $S$ and a line bundle $L$ on $S$, we generalize Levine and Pandharipande's construction to pairs of line bundles on surfaces. Let $L_{i}$ be line bundles on $X_{i}$, we call

$$
\left[X_{0}, L_{0}\right]-\left[X_{1}, L_{1}\right]-\left[X_{2}, L_{2}\right]+\left[X_{3}, L_{3}\right]
$$

an double point relation if $\left[X_{0}\right]-\left[X_{1}\right]-\left[X_{2}\right]+\left[X_{3}\right]$ is a double point relation, and there exists a line bundle $\mathcal{L}$ on $\mathcal{X}$ such that $L_{i}=\left.\mathcal{L}\right|_{X_{i}}$ for $i=0,1,2$; $L_{3}=\eta^{*}\left(\left.\mathcal{L}\right|_{D}\right)$ where $\eta: X_{3} \rightarrow D$ is the projection.

In $[\mathbf{L P}]$, Levine and Pandharipande defined the algebraic cobordism group of surfaces and line bundles $\omega_{2,1}$ to be the vector space over $\mathbb{Q}$ spanned by all pairs $[S, L]$ modulo all double point relations. The subscript $(2,1)$ captures the dimension of surfaces and the rank of line bundles. In Section 2, we prove $\omega_{2,1}$ is a four-dimensional vector space over $\mathbb{Q}$ and $\left(L^{2}, L K, c_{1}(S)^{2}\right.$, $\left.c_{2}(S)\right)$ induces the isomorphism from $\omega_{2,1}$ to $\mathbb{Q}^{4}$. Consequently, the class of $[S, L]$ in $\omega_{2,1}$ is linear in $L^{2}, L K, c_{1}(S)^{2}$ and $c_{2}(S)$. Two important bases of $\omega_{2,1}$ are

$$
\begin{aligned}
& \left\{\left[\mathbb{P}^{2}, \mathcal{O}\right],\left[\mathbb{P}^{2}, \mathcal{O}(1)\right],\left[\mathbb{P}^{1} \times \mathbb{P}^{1}, \mathcal{O}\right],\left[\mathbb{P}^{1} \times \mathbb{P}^{1}, \mathcal{O}(1,0)\right]\right\} \quad \text { and } \\
& \left\{\left[\mathbb{P}^{2}, \mathcal{O}\right],\left[\mathbb{P}^{2}, \mathcal{O}(1)\right],\left[S_{1}, L_{1}\right],\left[S_{2}, L_{2}\right]\right\}
\end{aligned}
$$

where $S_{i}$ are K3 surfaces and $L_{i}$ are primitive classes on $S_{i}$ with $L_{1}^{2} \neq L_{2}^{2}$.

In general, for pairs of vector bundles of rank $k$ on smooth projective scheme of dimension $n$, one can define the algebraic cobordism group $\omega_{n, k}$. 
Lee and Pandharipande $[\mathbf{L e e P}]$ have studied the structure of $\omega_{n, k}$. Our result about $\omega_{2,1}$, although worked out independently, is a special case of $[$ LeeP $]$.

Another important ingredient of our proof is the enumerative number $d_{r}(S, L)$. This number is defined by Göttsche $([\mathbf{G} \ddot{\mathbf{o}}])$ as the intersection number of a closed subscheme $W^{3 r}$ of $S^{[3 r]}$ and the $2 r^{t h}$ Chern class of the tautological bundle of $L$ on $S^{[3 r]}$, i.e.

$$
d_{r}(S, L)=\int_{W^{3 r}} c_{2 r}\left(L^{[3 r]}\right) .
$$

He proved that when $L$ is $(5 r-1)$-very ample, $d_{r}(S, L)$ equals the number of $r$-nodal curves in $[S, L]$. Although it is difficult to compute $d_{r}(S, L)$ directly, we believe it is the correct object to investigate for two reasons.

First, when a pair of smooth surface and ample line bundle degenerates to the singular fiber, ampleness of the line bundle is usually not preserved. Without ampleness, the universal polynomial $T_{r}\left(L^{2}, L K, c_{1}(S)^{2}, c_{2}(S)\right)$ do not necessarily equal to the number of $r$-nodal curves in $[S, L]$. On the contrary, the numbers $d_{r}(S, L)$ can be defined for every line bundle, even if it is not ample. In the end, we will show that for every pair $[S, L], d_{r}(S, L)$ is the universal polynomial $T_{r}\left(L^{2}, L K, c_{1}(S)^{2}, c_{2}(S)\right)$.

Second, since $d_{r}(S, L)$ is defined as an intersection number, it has many good properties under degeneration. Suppose $\pi: \mathcal{X} \rightarrow \mathbb{P}^{1}$ defines a double point relation and $U$ is a Zariski open set of $\mathbb{P}^{1}$ such that all fibers are smooth except $\pi^{-1}(\infty)$. Jun Li and Baosen Wu $[\mathbf{L W}]$ constructed a moduli stack $\mathcal{X}^{[n]} \rightarrow U$, which is the moduli stack of rank one stable relative ideal sheaves in the family $\mathcal{X}_{U}=\mathcal{X} \times_{\mathbb{P}^{1}} U \rightarrow U$. For each integer $n$, the moduli stack $\mathcal{X}^{[n]} \rightarrow U$ can be viewed as a family of Hilbert schemes of $n$ points on fibers. The generic fiber of $\mathcal{X}^{[n]} \rightarrow U$ at $\infty \neq t \in U$ is the Hilbert scheme $X_{t}^{[n]}$ and the special fiber is the union of products of relative Hilbert schemes

$$
\bigcup_{k=0}^{n}\left(X_{1} / D\right)^{[k]} \times\left(X_{2} / D\right)^{[n-k]} .
$$

For each $r \in \mathbb{N}$, recall $d_{r}(S, L)$ is defined to be $\int_{W^{3 r}} c_{2 r}\left(L^{[3 r]}\right)$. The closed subscheme $W^{3 r}$ in $S^{[3 r]}$ can be extended globally to a family of closed subschemes $\mathcal{W}^{3 r}$ in $\mathcal{X}^{[3 r]}$. In addition, if $\mathcal{L}$ is a line bundle on $\mathcal{X}$, then its restriction on $U$ similarly defines a tautological bundle $\mathcal{L}^{[n]}$ on $\mathcal{X}^{[n]}$ for each $n$. Thus on $\mathcal{X}^{[3 r]}$, the intersection of $c_{2 r}\left(\mathcal{L}^{[3 r]}\right)$ and $\mathcal{W}^{[3 r]}$ defines a family of zero cycles. Consider a double point relation $\left[X_{0}, L_{0}\right]-\left[X_{1}, L_{1}\right]-\left[X_{2}, L_{2}\right]+$ $\left[X_{3}, L_{3}\right]$. By rational equivalence of the fibers of $W^{3 r}$ over 0 and $\infty$, we show the generating function

$$
\phi(S, L)(x)=\sum_{r=0}^{\infty} d_{r}(S, L) x^{r}
$$


satisfies the following degeneration formula:

$$
\phi\left(X_{0}, L_{0}\right)=\phi\left(X_{1} / D, L_{1}\right) \phi\left(X_{2} / D, L_{2}\right),
$$

where $\phi\left(X_{i} / D, L_{i}\right)$ is the "relative" generating function.

The relation of absolute generating function $\phi$ can be computed by applying the degeneration formula on several families to eliminate the relative functions, which is

$$
\phi\left(X_{0}, L_{0}\right)=\frac{\phi\left(X_{1}, L_{1}\right) \phi\left(X_{2}, L_{2}\right)}{\phi\left(X_{3}, L_{3}\right)} .
$$

Thus $\phi$ induces a group homomorphism from the algebraic cobordism group $\omega_{2,1}$ to $\left(\mathbb{Q}[[x]]^{\times}, \cdot\right)$. Consequently, Theorem 1.3 is proved and $d_{r}(S, L)$ equals the universal polynomial $T_{r}\left(L^{2}, L K, c_{1}(S)^{2}, c_{2}(S)\right)$ for all smooth projective surfaces $S$ and line bundles $L$. Since $d_{r}(S, L)$ equals the number of $r$-nodal curves when $L$ is $(5 r-1)$-very ample, Theorem 1.1 is proved as a corollary.

The generating function in Theorem 1.2 is

$$
\gamma(S, L)(q)=\sum_{r \in \mathbb{Z}} T_{r}\left(L^{2}, L K, c_{1}(S)^{2}, c_{2}(S)\right)\left(D G_{2}(\tau)\right)^{r}=\phi(S, L)\left(D G_{2}\right) .
$$

Therefore this generating function also induces a homomorphism from $\omega_{2,1}$ to $\mathbb{Q}[[q]]^{\times}$. Since Bryan and Leung $[\mathbf{B L}]$ have computed $\gamma(S, L)(q)$ on generic K3 surfaces and primitive classes, we shall use a different basis

$$
\left\{\left[\mathbb{P}^{2}, \mathcal{O}\right],\left[\mathbb{P}^{2}, \mathcal{O}(1)\right],\left[S_{1}, L_{1}\right],\left[S_{2}, L_{2}\right]\right\}
$$

of $\omega_{2,1}$, where $S_{i}$ are K3 surfaces and $L_{i}$ are primitive classes on $S_{i}$ with $L_{1}^{2} \neq L_{2}^{2}$. By Theorem 1.3, $\gamma(S, L)(q)$ is a weighted product of $\gamma\left(\mathbb{P}^{2}, \mathcal{O}\right)$, $\gamma\left(\mathbb{P}^{2}, \mathcal{O}(1)\right), \gamma\left(S_{1}, L_{1}\right)$ and $\gamma\left(S_{2}, L_{2}\right)$. This proves Theorem 1.2. See Section 4 for more details about proofs and computation.

1.5. Outline. In Section 2 we briefly review the construction of two algebraic cobordism theories. In particular we construct the algebraic cobordism group $\omega_{2,1}$ and study its structure.

Section 3 is dedicated to the enumerative number $d_{r}(S, L)$ and its generating function $\phi(S, L)(x)$. The main result in this section is a degeneration formula about $\phi(S, L)(x)$ for pairs satisfying a double point relation.

Finally, in Section 4 we combine the techniques developed in Sections 2 and 3 to prove Theorems 1.1, 1.2 and 1.3. We express the infinite series $A_{i}$ and $B_{i}$ as the weighted product of generating functions on $\mathbb{P}^{2}, \mathbb{P}^{1} \times \mathbb{P}^{1}$, and K3 surfaces.

1.6. Acknowledgments. I am indebted to my advisor Jun Li for his supervision, advice, and guidance. His contribution of time, ideas, and patience is invaluable. Special thanks also to Ravi Vakil for suggestions and help in all aspects. Moreover, I thank Lothar Göttsche for the explanation of his approach and Rahul Pandharipande for useful suggestions. I am also grateful to Professor Shing-Tung Yau for his support and encouragements. 


\subsection{Notation and Convention.}

(1) All surfaces are assumed to be complex, projective, smooth and algebraic.

(2) An $r$-nodal curve is a reduced connected curve that has exactly $r$ nodes and no other types of singularity.

(3) We always denote by $[S, L]$ a pair of a smooth projective surface $S$ and a line bundle $L$ on $S$.

(4) The number of $r$-nodal curves in a pair $[S, L]$ means the number of $r$-nodal curves in a generic $r$-dimensional linear system of $|L|$ on $S$.

\section{Algebraic Cobordism}

2.1. Introduction. In this section, we assume $k$ be a field of characteristic 0. Let $\mathbf{S c h}_{k}$ denote the category of separated schemes of finite type over $k$ and $\mathbf{S m}_{k}$ denote its full subcategory consisting of smooth quasi-projective $k$-schemes.

The theory of algebraic cobordism $\Omega^{*}$ was first constructed by Levine and Morel $[\mathbf{L M}]$, which lifts the complex cobordism theory to algebraic varieties over a field of characteristic zero. They proved that $\Omega^{*}$ is a universal oriented cohomology theory on $\mathbf{S m}_{k}$ endowed with a universal formal group law. Other examples of oriented cohomology include the Chow ring $X \rightarrow \mathbf{C H}^{*}(X)$, the Grothendieck group of coherent sheaves joint with $\beta$, $\beta^{-1}: X \rightarrow K^{0}(X)\left[\beta, \beta^{-1}\right]$. The universality of $\Omega^{*}$ implies there are induced morphisms of oriented cohomology theory

$$
\begin{aligned}
\Omega^{*} \otimes_{\mathbb{L}^{*}} \mathbb{Z} & \rightarrow \mathbf{C H}^{*} \\
\Omega^{*} \otimes_{\mathbb{L}^{*}} \mathbb{Z}\left[\beta, \beta^{-1}\right] & \rightarrow K^{0}\left[\beta, \beta^{-1}\right]
\end{aligned}
$$

which are in fact isomorphisms. Thus $\mathbf{C H}^{*}$ and $K^{0}\left[\beta, \beta^{-1}\right]$ can be obtained by changing the formal group laws on $\Omega^{*}$, which is done by tensoring factors $\mathbb{Z}$ and $\mathbb{Z}\left[\beta, \beta^{-1}\right]$.

When Levine and Morel constructed $\Omega^{*}$, they first identified the homology theory $\Omega_{*}$ on $\mathbf{S c h}_{k}$ and identify two theories by $\Omega^{n}(X)=\bigoplus \Omega_{d_{\alpha}-n}\left(X_{\alpha}\right)$ where $X=\cup X_{\alpha}$ is the union of irreducible components and the dimension of $\left(X_{\alpha}\right)$ is $d_{\alpha} . \Omega_{*}(X)$ are generated by cobordism cycles $[f: Y \rightarrow$ $\left.X, L_{1}, L_{2}, \ldots, L_{r}\right]$ where $Y$ is in $\mathbf{S m}_{k}, f$ is projective and $L_{i}$ 's are line bundles over $Y$. Define the degree of such a cobordism cycle to be $\operatorname{dim}_{k}(Y)-r$. Then $\Omega_{*}(X)$ is defined as the free abelian group generated by cobordism cycles modulo some natural relations. Briefing speaking, if $L$ is a line bundle on $X$, we hope to have

$$
\begin{aligned}
c_{1}(L)\left[f: Y \rightarrow X, L_{1}, L_{2}, \ldots, L_{r}\right] & :=\left[f: Y \rightarrow X, L_{1}, L_{2}, \ldots, L_{r}, f^{*} L\right] \\
& =\left[f \circ i: Z \rightarrow X, L_{1}, L_{2}, \ldots, L_{r}\right],
\end{aligned}
$$

where $i: Z \rightarrow Y$ is a closed subvariety of the zeroes of $s$ and $s$ is a section of $L$ transversal to the zero section. Thus the relations in $\Omega_{*}(X)$ are imposed to make the equality always hold, and the first Chern class satisfies projective 
bundle formula, homotopy invariance, nilpotence, and formal group law axioms.

Instead of dealing with a large number of generators and relations, Levine and Pandharipande constructed another algebraic cobordism theory $\omega_{*}$ by allowing only one type of relation called the double point degeneration. More precisely, they consider projective morphisms

$$
\pi: Y \rightarrow X \times \mathbb{P}^{1}
$$

and composition

$$
\pi_{2}=p_{2} \circ \pi: Y \rightarrow \mathbb{P}^{1}
$$

where $X \in \mathbf{S c h}_{k}$ and $Y$ is a smooth quasi-projective scheme of pure dimension. Assuming $\pi_{2}^{-1}(\infty)=A \cup B$, where $A$ and $B$ are smooth Cartier divisors intersecting transversely at $D=A \cap B$,

$$
X_{3}:=\mathbb{P}\left(\mathcal{O}_{D} \otimes N_{A / D}\right) \cong \mathbb{P}\left(N_{B / D} \otimes \mathcal{O}_{D}\right)
$$

is a $\mathbb{P}^{1}$ bundle over $D$, and $Y_{0}$ is the fiber of $\pi_{2}$ over 0 . The double point relation over $X$ defined by $\pi$ is

$$
\left[Y_{0} \rightarrow X\right]-[A \rightarrow X]-[B \rightarrow X]+\left[X_{3} \rightarrow X\right]
$$

Let $\mathcal{M}(X)^{+}$be the free additive group generated by $[M \rightarrow X]$, where $M$ is a quasi-projective smooth scheme and the morphism is projective. Denote $\mathcal{R}(X)$ to be the subgroup generated by all double point relations over $X$ and $\omega_{*}(X)=\mathcal{M}(X)^{+} / \mathcal{R}(X)$. Then mapping $[M \rightarrow X]$ to itself descends to a morphism between two algebraic cobordism theories:

THEOREM $2.1([\mathbf{L P}])$. There is a canonical isomorphism

$$
\omega_{*}(X) \cong \Omega_{*}(X) .
$$

As a result, $\omega_{*}$ satisfies all properties of $\Omega_{*}$. In particular, if $k$ is a field of characteristic 0 , we denote $\Omega(k)$ to be $\Omega_{*}(\operatorname{Spec} k)$ and $\omega_{*}(k)$ to be $\omega_{*}($ Spec $k)$ in short. Levine and Morel showed that $\Omega_{*}(k)$ is isomorphic to the Lazard ring $\mathbb{L}_{*}$; consequently also isomorphic to $\omega_{*}(k)$. Furthermore, if $k$ admits an embedding to $\mathbb{C}$, then

$$
\begin{aligned}
& \omega_{*}(k) \rightarrow \quad \Omega_{*}(k) \rightarrow \quad M U_{2 *} \cong \mathbb{L}_{*} \\
& {[X \rightarrow \text { Speck }] \rightarrow[X \rightarrow \text { Speck }] \rightarrow\left[X \times_{\text {Speck }} \text { Spec } \mathbb{C}\right]}
\end{aligned}
$$

are isomorphisms, where the last morphism is Quillen's isomorphism. Since $M U_{2 *} \otimes_{\mathbb{Z}} \mathbb{Q} \cong \mathbb{L}_{*} \otimes_{\mathbb{Z}} \mathbb{Q}$ has a basis formed by products of projective spaces, it follows that

Corollary 2.2 .

$$
\Omega_{*}(k) \otimes_{\mathbb{Z}} \mathbb{Q} \cong \omega_{*}(k) \otimes_{\mathbb{Z}} \mathbb{Q} \cong \bigoplus_{\lambda=\left(\lambda_{1}, \ldots, \lambda_{r}\right)} \mathbb{Q}\left[\mathbb{P}^{\lambda_{1}} \times \cdots \times \mathbb{P}^{\lambda_{r}}\right]
$$


where the index $\lambda$ belongs to $\mathbb{N}^{r}$ for some positive integer $r$. Moreover, the class of a smooth scheme $X$ is uniquely determined by all Chern numbers of $X$, in the same way as complex cobordism theory

In particular, $\omega_{2}(k)$ is generated by $\mathbb{P}^{2}$ and $\mathbb{P}^{1} \times \mathbb{P}^{1}$ over $\mathbb{Q}$, i.e. all smooth projective surfaces, up to a multiple, can be degenerated to the sum of $\mathbb{P}^{2}$ and $\mathbb{P}^{1} \times \mathbb{P}^{1}$ using double point relations. In addition, the coefficients of the sum are uniquely determined by the Chern numbers of the surface.

For our purpose of counting nodal curves, degenerating surfaces alone are not sufficient. Instead, we consider the degeneration of pairs $[S, L]$ where $S$ is a smooth projective surface and $L$ is a line bundle on $S$.

Definition 2.1. Suppose $\left[X_{i}, L_{i}\right]$ are pairs of smooth projective surfaces over $k$ and line bundles for $i=0,1,2,3$. The double point relation for such pairs is defined by

$$
\left[X_{0}, L_{0}\right]=\left[X_{1}, L_{1}\right]+\left[X_{2}, L_{2}\right]-\left[X_{3}, L_{3}\right]
$$

with the assumption that there exists a flat family of surfaces $\pi: \mathcal{X} \rightarrow \mathbb{P}^{1}$ and a line bundle $\mathcal{L}$ on $\mathcal{X}$ which satisfies the following properties:

(1) the family $\pi: \mathcal{X} \rightarrow \mathbb{P}^{1}$ gives a double point relation on surfaces;

(2) $L_{i}$ is the restriction of $\mathcal{L}$ on $X_{i}$ for $i=0,1,2$;

(3) $L_{3}$ is the pullback of $\left.\mathcal{L}\right|_{D}$ via the projection $X_{3} \rightarrow D$.

Remark. We use $X_{1} / D$ and $X_{2} / D$ to emphasize the divisor $D$ when discussing the relative geometry on $X_{i}$.

Let $M(k)$ be the $\mathbb{Q}$-vector space ${ }^{2}$ spanned by pairs of smooth projective surfaces and line bundles, and let $R(k)$ be the subgroup spanned by all double point relations for pairs. We define the algebraic cobordism group of surfaces and line bundles to be

$$
\omega_{2,1}(k)=M(k) / R(k) .
$$

Because the double point relation on surfaces can be viewed as a double point relation on pairs of surfaces with trivial line bundle, $\omega_{2}(k)$ naturally embeds into $\omega_{2,1}(k)$

By definition, $\omega_{2,1}(k)$ is a vector space over $\mathbb{Q}$. We are interested in finding its dimension, bases, and invariants of this degeneration theory.

Proposition 2.3. Let $S$ be a smooth projective surface over $k$ and $L$ be a line bundle on $S$, then

$$
[S, L]=a_{1}\left[\mathbb{P}^{2}, \mathcal{O}\right]+a_{2}\left[\mathbb{P}^{2}, \mathcal{O}(1)\right]+a_{3}\left[\mathbb{P}^{1} \times \mathbb{P}^{1}, \mathcal{O}\right]+a_{4}\left[\mathbb{P}^{1} \times \mathbb{P}^{1}, \mathcal{O}(1,0)\right]
$$

\footnotetext{
${ }^{2}$ We use $\mathbb{Q}$ here because it is enough for our purpose and for simplicity. In general, the coefficients can be $\mathbb{Z}$ as discussed in $[\mathbf{L P}]$.
} 
in $\omega_{2,1}(k)$, where

$$
\begin{array}{ll}
a_{1}=-L^{2}+\frac{c_{1}(S)^{2}+c_{2}(S)}{3}-c_{2}(S), & a_{2}=L^{2}, \\
a_{3}=L^{2}+\frac{L K+L^{2}}{2}-\frac{c_{1}(S)^{2}+c_{2}(S)}{4}+c_{2}(S), & a_{4}=-L^{2}-\frac{L K+L^{2}}{2} .
\end{array}
$$

In other words, $\omega_{2,1}(k)$ is a four dimensional vector space spanned by four elements $\left[\mathbb{P}^{2}, \mathcal{O}\right],\left[\mathbb{P}^{2}, \mathcal{O}(1)\right],\left[\mathbb{P}^{1} \times \mathbb{P}^{1}, \mathcal{O}\right]$ and $\left[\mathbb{P}^{1} \times \mathbb{P}^{1}, \mathcal{O}(1,0)\right]$. Moreover, $\left(L^{2}, L K, c_{1}(S)^{2}, c_{2}(S)\right)$ defines an isomorphism from $\omega_{2,1}(k)$ to $\mathbb{Q}^{4}$.

Remark. Proposition 2.3 implies the class of $[S, L]$ in $\omega_{2,1}$ is uniquely determined by degree two Chern polynomials of $c_{1}(S), c_{2}(S)$ and $c_{1}(L)$ which is a generalization of the case of schemes. Noether's formula states

$$
\chi\left(\mathcal{O}_{S}\right)=\frac{1}{12}\left(c_{1}(S)^{2}+c_{2}(S)\right)
$$

and thus $c_{1}(S)^{2}+c_{2}(S)$ is divisible by three and four. In addition, the Riemann-Roch formula for $L$ is

$$
\chi(L)=\chi\left(\mathcal{O}_{S}\right)+\frac{1}{2}\left(L^{2}-L K\right),
$$

and therefore $L^{2}-L K$ is divisible by two. Therefore all coefficients in (2.2) are integers.

In $[\mathbf{T z}]$, we give a elementary proof of Proposition 2.3, which starts with the following observation:

Lemma 2.4. Let $C$ be a smooth curve in $S, L$ be a line bundle on $S, N$ be the normal bundle of $C$ in $S$ and $\eta: \mathbb{P}_{C}\left(N \oplus \mathcal{O}_{C}\right) \rightarrow C$ be the structure map. Then

$$
[S, L]=\left[S, L \otimes \mathcal{O}_{S}(-C)\right]+\text { pairs on } \mathbb{P}_{C}\left(N \oplus \mathcal{O}_{C}\right)
$$

in $\omega_{2,1}(k)$.

Consequently, we are allowed to add effective divisors freely to the line bundle. The remaining terms are on ruled surfaces which have been classified. As a result Proposition 2.3 can be proved in the following steps.

(1) Write $L$ as the $\mathcal{O}\left(D_{1}-D_{2}\right)$, where $\mathcal{O}\left(D_{i}\right)$ are very ample and $D_{i}$ are smooth divisors. Then our observation implies $[S, L]$ is the sum of $[S, \mathcal{O}]$ plus pairs on ruled surfaces in the form $\mathbb{P}_{C}\left(N \oplus \mathcal{O}_{C}\right)$.

(2) Degeneration of $[S, \mathcal{O}]$ is the same as degenerating $[S]$ in $\omega_{2}(k)$, thus $[S, \mathcal{O}]$ can be written as the sum of several $\left[\mathbb{P}^{2}, \mathcal{O}\right]$ and $\left[\mathbb{P}^{1} \times \mathbb{P}^{1}, \mathcal{O}\right]$.

(3) Pairs on $\mathbb{P}_{C}\left(N \ominus \mathcal{O}_{C}\right)$ can be further simplified by modifying the line bundle $N$ and degenerating the base curve $C$. Then we write explicit double point relations to conclude these pairs are generated by $\left[\mathbb{P}^{2}, \mathcal{O}(1)\right],\left[\mathbb{P}^{1} \times \mathbb{P}^{1}, \mathcal{O}\right]$ and $\left[\mathbb{P}^{1} \times \mathbb{P}^{1}, \mathcal{O}(1,0)\right]$. 
(4) Finally, we show $L^{2}, L K, c_{1}(S)^{2}$ and $c_{2}(S)$ are preserved in double point relations, thus are invariants of $\omega_{2,1}(k)$. It implies $\omega_{2,1}(k)$ has four generators and four independent invariants, so the generators is actually a basis of $\omega_{2,1}(k)$.

(5) Since $L^{2}, L K, c_{1}(S)^{2}$ and $c_{2}(S)$ are invariants of $\omega_{2,1}(k)$, it can be used to compute the coefficients of equation 2.2 .

\section{REMARK.}

(1) From the proof of Proposition 2.3, we know a set of four elements

$$
\left\{\left[S_{i}, L_{i}\right] \mid i=1, \ldots, 4\right\}
$$

is a basis of $\omega_{2,1}$ if and only if the four vectors

$$
\left\{\left(L_{i}^{2}, L_{i} K_{S_{i}}, c_{1}\left(S_{i}\right)^{2}, c_{2}\left(S_{i}\right)\right) \mid i=1, \ldots, 4\right\}
$$

are linearly independent over $\mathbb{Q}$.

(2) The sets $\left(L^{2}, L K, c_{1}(S)^{2}, c_{2}(S)\right)$ and $\left(L K, \chi(L), \chi(\mathcal{O}), K^{2}\right)$ determine each other, thus $\left(L K, \chi(L), \chi(\mathcal{O}), K^{2}\right)$ is also a set of invariants of $\omega_{2,1}$. As a result, if for a set of four elements in $\omega_{2,1}$, the corresponding vectors $\left(L K, \chi(L), \chi(\mathcal{O}), K^{2}\right)$ are linearly independent, then the set is a basis of $\omega_{2,1}$.

(3) If $S_{1}, S_{2}$ are two K3 surfaces and $L_{i}$ are primitive classes on $S_{i}$ respectively, the four numbers $\left(L K, \chi(L), \chi(\mathcal{O}), K^{2}\right)$ of

$$
\begin{gathered}
\mathfrak{B}=\left\{\left[\mathbb{P}^{2}, \mathcal{O}\right],\left[\mathbb{P}^{2}, \mathcal{O}(1)\right],\left[S_{1}, L_{1}\right],\left[S_{2}, L_{2}\right]\right\} \quad \text { are } \\
(0,1,1,9),(-3,3,1,9),\left(0,2+\frac{L_{1}^{2}}{2}, 2,0\right),\left(0,2+\frac{L_{2}^{2}}{2}, 2,0\right) .
\end{gathered}
$$

It follows that $\mathfrak{B}$ is a basis if and only if $L_{1}^{2} \neq L_{2}^{2}$.

In general, the algebraic cobordism theory $\omega_{n, r}$ for smooth schemes of dimension $n$ and vector bundles of rank $r$ have been constructed analogously by Y.P. Lee and Pandharipande $[\mathbf{L e e P}]$ for all nonnegative $n, r$. They proved that the invariants of $\omega_{n, r}$ are all possible degree $n$ monomials of the Chern classes of the scheme and the vector bundle. Furthermore, there exists a basis consisting of products of projective spaces pairs with direct sum of selected pullback of $\mathcal{O}(1)$ 's.

\section{Degeneration Formula}

3.1. The enumerative number $d_{r}(S, L)$. In this section we use the enumerative number $d_{r}(S, L)$ to study the number of nodal curves. This number $d_{r}(S, L)$ was introduced by Göttsche [Gö] for pairs of smooth projective surface and line bundle $[S, L]$, and he proved that $d_{r}(S, L)$ equals the number of $r$-nodal curves on $[S, L]$ if $L$ is $(5 r-1)$-very ample. The goal of 
this section is to derive a degeneration formula for the generating function

$$
\phi(S, L)(x)=\sum_{r=0}^{\infty} d_{r}(S, L) x^{r}
$$

for pairs satisfying double point relations.

Let $S^{[n]}$ be the Hilbert scheme of $n$ points on $S$, and let $Z_{n} \subset S \times S^{[n]}$ be the universal closed subscheme with projections

$$
p_{n}: Z_{n} \rightarrow S, q_{n}: Z_{n} \rightarrow S^{[n]} .
$$

Define $L^{[n]}=\left(q_{n}\right)_{*}\left(p_{n}\right)^{*} L$. Because $q_{n}$ is finite and flat, $L^{[n]}$ is a vector bundle of rank $n$ on $S^{[n]}$. Göttsche [Gö] suggested the following approach, which interprets the number of nodal curves as intersection numbers on Hilbert schemes:

Definition 3.1 ([Gö], definition 5.1). Let $W_{0}^{3 r}$ be the locally closed subset

$$
\left\{\coprod_{i=1}^{r} \operatorname{Spec}\left(\mathcal{O}_{S, x_{i}} / m_{S, x_{i}}^{2}\right) \mid x_{i} \text { are distinct closed points on } S\right\}
$$

and $W^{3 r} \subset S^{[3 r]}$ be the closure of $W_{0}^{3 r}$ (with the reduced induced structure). It is easy to see that $W^{3 r}$ is birational to $S^{[r]}$. Define

$$
d_{r}(S, L)=\int_{W^{3 r}} c_{2 r}\left(L^{[3 r]}\right) .
$$

For simplicity, define $d_{0}(S, L)=1$ because the number of 0 -nodal curves, which by definition are smooth, in a zero-dimensional linear system is one.

Definition 3.2. We call a line bundle $L k$-very ample if for every zerodimensional subscheme $\xi \subset S$ of length $k+1$, the natural map $H^{0}(S, L) \rightarrow$ $H^{0}\left(\xi, L \otimes \mathcal{O}_{\xi}\right)$ is surjective.

If $L$ and $M$ are very ample, then $L^{\otimes k} \otimes M^{\otimes l}$ is $(k+l)$-very ample. In particular, very ampleness implies 1-very ampleness.

We quote a result of Göttsche below:

Proposition 3.1 ([Gö], proposition 5.2). Assume $S$ is a smooth algebraic surface and $L$ is a $(5 r-1)$-very ample line bundle on $S$, then a general $r$-dimensional sublinear system $V \subset|L|$ contains $d_{r}(S, L)$ curves with precisely $r$-nodes as singularities.

3.2. Degeneration Formula. In Section 1.4 we explained why $d_{r}$ $(S, L)$ behaves better than the actual number of nodal curves in flat families. The goal of this section is to derive a degeneration formula for $d_{r}(S, L)$. More precisely, we will show that if

$$
\left[X_{0}, L_{0}\right]=\left[X_{1}, L_{1}\right]+\left[X_{2}, L_{2}\right]-\left[X_{3}, L_{3}\right]
$$


is a double point relation, then then number of nodal curves on $\left[X_{0}, L_{0}\right]$ can be determined by the numbers on $\left[X_{1}, L_{1}\right],\left[X_{2}, L_{2}\right]$ and $\left[X_{3}, L_{3}\right]$. Thus it is necessary to treat the number of curves with varied number of nodes together by considering the generating function

$$
\phi(S, L)(x)=\sum_{r=0}^{\infty} d_{r}(S, L) x^{r} .
$$

The following theorem is the main result of this section:

Proposition 3.2. Suppose $\left[X_{0}, L_{0}\right]=\left[X_{1}, L_{1}\right]+\left[X_{2}, L_{2}\right]-\left[X_{3}, L_{3}\right]$ is a double point relation. Then

$$
\phi\left(X_{0}, L_{0}\right)=\frac{\phi\left(X_{1}, L_{1}\right) \cdot \phi\left(X_{2}, L_{2}\right)}{\phi\left(X_{3}, L_{3}\right)} .
$$

In other words, $\phi$ is a homomorphism from $\omega_{2,1}$ to $\left(\mathbb{Q}[[x]]^{\times}, \cdot\right)$.

3.3. Moduli stack of relative ideal sheaves. The key tool is Jun $\mathrm{Li}$ and Baosen Wu's $[\mathbf{L W}]$ construction of the moduli stack of stable relative ideal sheaves. Similar construction has been applied successfully to prove the degeneration formulas of Gromov-Witten invariants [Li] and of DonalsdonThomas invariants $[\mathbf{L W}]$. Our case is even simpler because we only consider families of surfaces and ideal sheaves on points on surfaces.

Let $\infty \in C$ be a specialized point and $\pi: X \rightarrow C$ be a flat projective family of schemes that satisfies

(1) $X$ is smooth and $\pi$ is smooth away from the fiber $\pi^{-1}(\infty)$;

(2) $\pi^{-1}(\infty)=: X_{1} \cup_{D} X_{2}$ is a union of two irreducible smooth components $X_{1}$ and $X_{2}$ which intersect transversally along a smooth divisor $D$.

In $[\mathbf{L W}], \mathrm{Li}$ and $\mathrm{Wu}$ defined the notion of a family of stable perfect ideal sheaves over $C$ and constructed $\mathfrak{I}_{X / C}^{\Gamma}$, the moduli space of stable perfect ideal sheaves of type $\Gamma$ of $X \rightarrow C$. To make $\mathfrak{I}_{X / C}^{\Gamma}$ a stack, one has to replace $X$ by new spaces $X[n]$ so that $X$ and $X[n]$ have the same smooth fiber $X_{t}$ when $t \neq \infty$. Over $\infty$, the fiber of $X[n]$ is a semistable model

$$
X[n]_{0}=X_{1} \cup \Delta_{1} \cup \Delta_{2} \cup \cdots \Delta_{n-1} \cup X_{2},
$$

where $\Delta_{i} \cong \mathbb{P}_{D}\left(\mathcal{O}_{D} \oplus N_{X_{1} / D}\right)$. The objects of $\mathfrak{I}_{X / C}^{\Gamma}$ are expanded relative pairs $(\mathcal{X} / S, \mathcal{I})$ which consists of a family $\mathcal{X}$ over a $C$-scheme $S$ and a family of stable ideal sheaves $\mathcal{I}$ of type $\Gamma$ on $\mathcal{X} / S$. The fibers $\mathcal{X}_{s}$ of the family $\mathcal{X} / S$ are required to be either smooth a fiber of $X / C$ or a semistable model $X[n]_{0}$ for some $n$. Under these settings, $\mathrm{Li}$ and $\mathrm{Wu}$ proved that the moduli space has many good properties:

THEOREM $3.3([\mathbf{L W}])$. The moduli stack $\mathfrak{I}_{X / C}^{\Gamma}$ is a separated and proper Deligne-Mumford stack of finite type over $C$. 
In our case, $\pi: X \rightarrow C$ is a family of surfaces and $\mathcal{I}$ is a family of ideal sheaves of zero-dimensional closed subschemes of length $n$. For $s \in S$, when $\mathcal{X}_{s}$ is a smooth fiber of $X / C, \mathcal{I}_{s}$ is automatically perfect and stable. If $\mathcal{X}_{s}$ is a semistable model $X[m]_{0}$, then the support of $\mathcal{I}_{s}$ can not lie on the singular loci of $X[m]_{0}$. Furthermore, every component $\Delta_{i}$ contains at least one point of the zero-set of $\mathcal{I}_{s}$ (otherwise this component can be contracted).

The resulted moduli space $\mathfrak{I}_{X / C}^{n}$ a separated and proper DeligneMumford stack of finite type over $C$, and the fibers of the projection $\pi^{[n]}$ : $\mathfrak{I}_{X / C}^{n} \rightarrow C$ can be described as follows:

(1) The fiber over $\infty$ is the union of products

$$
\cup_{k=0}^{n}\left(X_{1} / D\right)^{[k]} \times\left(X_{2} / D\right)^{[n-k]}
$$

for all possible $n \geq k \geq 0$. $\left(X_{i} / D\right)^{\left[n_{i}\right]}$ are the moduli spaces of stable relative ideal sheaves of $n_{i}$ points on $X_{i} / D .\left(X_{i} / D\right)^{[0]}=p t$. They are also separated and proper Deligne-Mumford stacks (see $[\mathbf{L W}])$. We denote this fiber by $\left(X_{1} \cup_{D} X_{2}\right)^{[n]}$.

(2) $\mathfrak{I}_{X / C}^{n}$ is smooth and $\pi^{[n]}$ is smooth away from the fiber over $\infty$.

(3) When $t \neq \infty$, the smooth fiber of $\mathfrak{I}_{X / C}^{n}$ over $t$ equals $X_{t}^{[n]}$, the Hilbert schemes of $n$ points on $X_{t}$.

Therefore, $\mathfrak{I}_{X / C}^{n}$ can also be viewed as a family of Hilbert schemes of $n$ points on $X / C$.

Suppose a family of surfaces $\mathcal{X} \rightarrow \mathbb{P}^{1}$ and line bundle $\mathcal{L}$ on $\mathcal{X}$ gives a double point relation of line bundles on surfaces. This family $\mathcal{X}$ may have multiple singular fibers and only the fiber of $\infty$ is required to be two components intersection transversally. Therefore $\mathrm{Li}$ and Wu's construction can be applied only on an open subset of $\mathbb{P}^{1}$.

Let $U$ be a Zariski open set of $\mathbb{P}^{1}$ obtained by deleting those points with singular fibers except $\infty$, i.e. set-theoretically,

$$
U=\left\{t \in \mathbb{P}^{1} \mid \text { the fiber }\left.\mathcal{X}\right|_{t} \text { is smooth }\right\} \cup\{\infty\} .
$$

Then $\pi_{U}: \mathcal{X}_{U}:=\mathcal{X} \times_{\mathbb{P}^{1}} U \rightarrow U$ is a family of surfaces with only one singular fiber $X_{\infty}$.

Write $\mathfrak{I}_{\mathcal{X}_{U} / U}^{n}$ as $\mathcal{X}^{[n]}$. There are also universal closed subschemes over $\mathcal{X}^{[n]},\left(X_{1} / D\right)^{[n]}$ and $\left(X_{2} / D\right)^{[n]}$ respectively. Similarly, one can define rank $n$ vector bundles $\mathcal{L}^{[n]}, L_{1}^{[n]}$ and $L_{2}^{[n]}$ on $\left(X_{1} / D\right)^{[n]}$ and $\left(X_{2} / D\right)^{[n]}$ respectively, and closed substacks $\mathcal{W}^{3 r}, W_{X_{0}}^{3 r}$ and $W_{X_{i} / D}^{3 r}$ for $i=1,2$ in $\mathcal{X}^{[3 r]}, X_{0}^{[3 r]}$ and $\left(X_{i} / D\right)^{[3 r]}$. Let

$$
d_{r}\left(X_{i} / D, L_{i}\right)=\int_{W_{X_{i} / D}^{3 r}} c_{2 r}\left(L_{i}^{[3 r]}\right), d_{0}\left(X_{i} / D, L_{i}\right)=1
$$


and

$$
\phi\left(X_{i} / D, L_{i}\right)=\sum_{r \geq 0} d_{r}\left(X_{i} / D, L_{i}\right) x^{r} .
$$

The 1-cycle $\int_{\mathcal{W}^{3 r}} c_{2 r}\left(\mathcal{L}^{[3 r]}\right)$ restricts to fibers of $\mathcal{X}^{[n]}$ over 0 and $\infty$ gives two rational equivalent 0 -cycles with the same degree. Therefore

Proposition 3.4. Suppose $\left[X_{0}, L_{1}\right]-\left[X_{1}, L_{1}\right]-\left[X_{2}, L_{2}\right]+\left[X_{3}, L_{3}\right]$ is a double point relation, then

$$
\phi\left(X_{0}, L_{0}\right)=\phi\left(X_{1} / D, L_{1}\right) \cdot \phi\left(X_{2} / D, L_{2}\right) .
$$

To obtain a formula for absolute generating function, the relative surfaces can be closed up by adding projective bundles.

Corollary 3.5. Let $[X, L]$ be a pair in $\omega_{2,1}$. Suppose $D$ is a smooth curve in $X, N$ is the normal bundle of $D$ in $X$ and $L \cdot D$ is the intersection number of $c_{1}(L)$ and $D$. Recall $\mathbb{P}_{N}:=\mathbb{P}_{D}\left(\mathcal{O}_{D} \oplus N\right)$, then

$$
\phi(X, L)=\phi(X / D, L) \cdot \phi\left(\mathbb{P}_{N} / D,(L \cdot D) f\right) .
$$

Now we are ready to prove Proposition 3.2

Proof of Proposition 3.2. Apply Corollary 3.5 to $X=X_{1}, X_{2}$ and $\mathbb{P}_{N}$. It is easy to check the embeddings of $D$ match perfectly and $\left(L_{i} \cdot D\right) f \cong$ $\left(L_{2} \cdot D\right) f \cong L_{3}$ on $\mathbb{P}_{N}$. Thus we obtain

$$
\begin{aligned}
\phi\left(X_{0}, L_{0}\right) & =\phi\left(X_{1} / D, L_{1}\right) \cdot \phi\left(X_{2} / D, L_{2}\right), \\
\phi\left(X_{1} / D, L_{1}\right) \cdot \phi\left(\mathbb{P}_{N} / D_{0}, L_{3}\right) & =\phi\left(X_{1}, L_{1}\right), \\
\phi\left(X_{2} / D, L_{2}\right) \cdot \phi\left(\mathbb{P}_{N} / D_{\infty}, L_{3}\right) & =\phi\left(X_{2}, L_{2}\right), \\
\phi\left(\mathbb{P}_{N}, L_{3}\right) & =\phi\left(\mathbb{P}_{N} / D_{0}, L_{3}\right) \cdot \phi\left(\mathbb{P}_{N} / D_{\infty}, L_{3}\right) .
\end{aligned}
$$

Then the theorem is proved by multiplying all equations.

\section{Universality Theorems and Generating functions}

4.1. Outline. In this Section, we will prove Theorems 1.1, 1.2 and 1.3 by combining the degeneration formula (Proposition 3.2) with the structure of algebraic cobordism group $\omega_{2,1}$ (Proposition 2.3).

Recall that for any smooth projective surface $S$ and line bundle $L$ on $S$, we defined and studied the enumerative number $d_{r}(S, L)$ and generating function

$$
\phi(S, L)(x)=\sum_{r=0}^{\infty} d_{r}(S, L) x^{r}
$$

in Section 3. By Proposition 3.2, this function $\phi$ induces a homomorphism from $\omega_{2,1}$ to $\left(\mathbb{Q}[[x]]^{\times}, \cdot\right)$. On the other hand, Proposition 2.3 proves that $\omega_{2,1}$ is four-dimensional and the only invariants are $L^{2}, L K, c_{1}(S)^{2}$ and $c_{2}(S)$. Combining these two results, we show that $\phi(S, L)$ only depends on these four topological numbers and has a multiplicative structure. 


\subsection{Proof of Theorems 1.3 and 1.1 .}

Proposition 4.1. There exist four series $A_{1}, A_{2}, A_{3}$ and $A_{4}$ in $\mathbb{Q}[[x]]^{\times}$ such that

$$
\phi(S, L)(x)=A_{1}^{L^{2}} A_{2}^{L K_{S}} A_{3}^{c_{1}(S)^{2}} A_{4}^{c_{2}(S)} .
$$

More explicitly,

$$
\begin{aligned}
& A_{1}(x)=\phi\left(\mathbb{P}^{2}, \mathcal{O}\right)^{-1} \phi\left(\mathbb{P}^{2}, \mathcal{O}(1)\right) \phi\left(\mathbb{P}^{1} \times \mathbb{P}^{1}, \mathcal{O}\right)^{\frac{3}{2}} \phi\left(\mathbb{P}^{1} \times \mathbb{P}^{1}, \mathcal{O}(1,0)\right)^{-\frac{3}{2}}, \\
& A_{2}(x)=\phi\left(\mathbb{P}^{1} \times \mathbb{P}^{1}, \mathcal{O}\right)^{\frac{1}{2}} \phi\left(\mathbb{P}^{1} \times \mathbb{P}^{1}, \mathcal{O}(1,0)\right)^{-\frac{1}{2}} \\
& A_{3}(x)=\phi\left(\mathbb{P}^{2}, \mathcal{O}\right)^{-\frac{1}{3}} \phi\left(\mathbb{P}^{1} \times \mathbb{P}^{1}, \mathcal{O}\right)^{-\frac{1}{4}} \\
& A_{4}(x)=\phi\left(\mathbb{P}^{2}, \mathcal{O}\right)^{-\frac{2}{3}} \phi\left(\mathbb{P}^{1} \times \mathbb{P}^{1}, \mathcal{O}\right)^{\frac{3}{4}}
\end{aligned}
$$

Proof. By Proposition 2.3, the class of $[S, L]$ in $\omega_{2,1}$ is

$$
[S, L]=a_{1}\left[\mathbb{P}^{2}, \mathcal{O}\right]+a_{2}\left[\mathbb{P}^{2}, \mathcal{O}(1)\right]+a_{3}\left[\mathbb{P}^{1} \times \mathbb{P}^{1}, \mathcal{O}\right]+a_{4}\left[\mathbb{P}^{1} \times \mathbb{P}^{1}, \mathcal{O}(1,0)\right]
$$

where

$$
\begin{array}{ll}
a_{1}=-L^{2}+\frac{c_{1}(S)^{2}+c_{2}(S)}{3}-c_{2}(S), & a_{2}=L^{2}, \\
a_{3}=L^{2}+\frac{L K+L^{2}}{2}-\frac{c_{1}(S)^{2}+c_{2}(S)}{4}+c_{2}(S), & a_{4}=-L^{2}-\frac{L K+L^{2}}{2} .
\end{array}
$$

Since the generating function $\phi$ is a homomorphism from $\omega_{2,1}$ to $\left(\mathbb{Q}[[x]]^{\times}, \cdot\right)$ (Proposition 3.2), we obtain

$$
\phi(S, L)(x)=\phi\left(\mathbb{P}^{2}, \mathcal{O}\right)^{a_{1}} \phi\left(\mathbb{P}^{2}, \mathcal{O}(1)\right)^{a_{2}} \phi\left(\mathbb{P}^{1} \times \mathbb{P}^{1}, \mathcal{O}\right)^{a_{3}} \phi\left(\mathbb{P}^{1} \times \mathbb{P}^{1}, \mathcal{O}(1,0)\right)^{a_{4}} .
$$

Plugging in the values of $a_{i}$ and grouping the functions according to $L^{2}$, $L K, c_{1}(S)^{2}$ and $c_{2}(S)$ complete the proof.

Another way to prove Proposition 4.1 is using the following diagram:

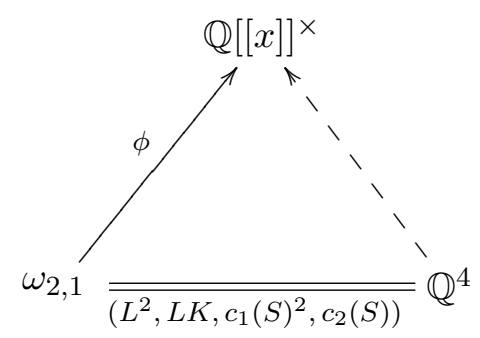

Since $\phi$ is a homomorphism and $\omega_{2,1} \rightarrow \mathbb{Q}^{4}$ is an isomorphism, they induce a homomorphism from $\mathbb{Q}^{4}$ to $\mathbb{Q}[[x]]^{\times}$. This implies $\phi(S, L)(x)$ is a universal power series in $L^{2}, L K, c_{1}(S)^{2}$ and $c_{2}(S)$. Furthermore, let the image of the standard basis $\mathbf{e}_{\mathbf{i}}$ of $\mathbb{Q}^{4}$ be $A_{i}$; then the commutative diagram implies

$$
\phi(S, L)(x)=A_{1}^{L^{2}} A_{2}^{L K_{S}} A_{3}^{c_{1}(S)^{2}} A_{4}^{c_{2}(S)} .
$$


REMARK. One can see the same proof is still valid if we replace

$$
\left\{\left[\mathbb{P}^{2}, \mathcal{O}\right],\left[\mathbb{P}^{2}, \mathcal{O}(1)\right],\left[\mathbb{P}^{1} \times \mathbb{P}^{1}, \mathcal{O}\right],\left[\mathbb{P}^{1} \times \mathbb{P}^{1}, \mathcal{O}(1,0)\right]\right\}
$$

by any basis of $\omega_{2,1}$. Thus the four series $A_{i}^{\prime} s$ can also be expressed in the generating function $\phi$ of any basis.

Corollary 4.2. The enumerative number $d_{r}(S, L)$ is a universal polynomial of degree $r$ in $L^{2}, L K, c_{1}(S)^{2}$ and $c_{2}(S)$ for all projective smooth surfaces $S$ and line bundles $L$ on $S$.

Proof. For all pairs $[S, L], d_{r}(S, L)$ is the coefficient of $x^{r}$ in

$$
\phi(S, L)(x)=A_{1}^{L^{2}} A_{2}^{L K_{S}} A_{3}^{c_{1}^{2}(S)} A_{4}^{c_{2}(S)} .
$$

After expanding the series, one can see that the coefficient of $x^{r}$ in $\phi(S, L)(x)$ is a universal polynomial of $\left(L^{2}, L K, c_{1}(S)^{2}, c_{2}(S)\right)$ of degree $r$.

Next, we prove Göttsche's conjecture:

THEOREM 1.1. For every integer $r \geq 0$, there exists a universal polynomial $T_{r}(x, y, z, t)$ of degree $r$ with the following property: given a smooth projective surface $S$ and $a(5 r-1)$-very ample (5-very ample if $r=1)$ line bundle $L$ on $S$, a general $r$-dimensional sublinear system of $|L|$ contains exactly $T_{r}\left(L^{2}, L K, c_{1}(S)^{2}, c_{2}(S)\right)$ r-nodal curves.

Proof. $d_{r}(S, L)$ is always a universal polynomial of degree $r$ for all line bundles $L$ on $S$. If $L$ is $(5 r-1)$-very ample, Proposition 3.1 shows $d_{r}(S, L)$ equals the number of $r$-nodal curves in $[S, L]$. Thus the number of $r$-nodal curves in $[S, L]$ is given by a universal polynomial of degree $r$ when $L$ is $(5 r-1)$-very ample.

Corollary 4.3. The generating functions $\phi(S, L)(x)$ and $T(S, L)(x)$ (defined in Section 1.3 as $\sum_{r=0}^{\infty} T_{r}\left(L^{2}, L K, c_{1}(S)^{2}, c_{2}(S)\right) x^{r}$ ) are equal.

TheOREM 1.3. There exist universal power series $A_{1}, A_{2}, A_{3}, A_{4}$ in $\mathbb{Q}[[x]]^{\times}$such that the generating function $T(S, L)$ has the form

$$
T(S, L)=A_{1}^{L^{2}} A_{2}^{L K_{S}} A_{3}^{c_{1}(S)^{2}} A_{4}^{c_{2}(S)} .
$$

Proof. This follows from Proposition 4.1 and Corollary 4.3.

4.3. Proof of the Göttsche-Yau-Zaslow Formula. Let $\tau$ be on the complex upper half plane, $q=e^{2 \pi i \tau}$. The Eisenstein series

$$
G_{2}(\tau):=\frac{-1}{24}+\sum_{n>0}\left(\sum_{d \mid n} d\right) q^{n}
$$

is a quasimodular form. Denote by $D$ the differential operator $D:=\frac{1}{2 \pi i} \frac{d}{d \tau}=$ $q \frac{d}{d q}$. Since the ring of quasimodular forms is closed under differentiation, 
$D G_{2}$ and $D^{2} G_{2}$ are also quasimodular. In addition, let

$$
\Delta(\tau)=q \prod_{k>0}\left(1-q^{k}\right)^{24}=\eta(\tau)^{24}
$$

where $\eta(\tau)$ is the Dedekind $\eta$ function, and $\Delta(\tau)$ is a modular form. We define the generating function

$$
\gamma(S, L)(q)=\sum_{r=0}^{\infty} T_{r}\left(L^{2}, L K, c_{1}(S)^{2}, c_{2}(S)\right)\left(D G_{2}(\tau)\right)^{r} .
$$

An equivalent form of Bryan and Leung's result ([BL], theorem 1.1) states that for a generic K3 surface $S$ and a primitive class $L$,

$$
\gamma(S, L)(q)=\frac{\left(D G_{2}(\tau) / q\right)^{\chi(L)}}{\Delta(\tau) D^{2} G_{2}(\tau) / q^{2}}
$$

More generally, the generating function $\gamma(S, L)(q)$ for all algebraic surfaces is given by

Theorem 1.2 (Göttsche-Yau-Zaslow Formula). There exist universal power series $B_{1}, B_{2}$ in $q$ such that

$$
\begin{gathered}
\sum_{r=0}^{\infty} T_{r}\left(L^{2}, L K, c_{1}(S)^{2}, c_{2}(S)\right)\left(D G_{2}(\tau)\right)^{r} \\
=\frac{\left(D G_{2}(\tau) / q\right)^{\chi(L)} B_{1}(q)^{K_{S}^{2}} B_{2}(q)^{L K_{S}}}{\left(\Delta(\tau) D^{2} G_{2}(\tau) / q^{2}\right)^{\chi\left(\mathcal{O}_{S}\right) / 2}} .
\end{gathered}
$$

Proof. Since $\left(K_{S}^{2}, L K_{S}, \chi(L), \chi\left(\mathcal{O}_{S}\right)\right)$ and $\quad\left(L^{2}, L K, c_{1}(S)^{2}, c_{2}(S)\right)$ determine each other linearly, $\phi(S, L)$ is multiplicative in $\left(K_{S}^{2}, L K_{S}, \chi(L)\right.$, $\left.\chi\left(\mathcal{O}_{S}\right)\right)$ and so as $\gamma(S, L)(q)=\phi(S, L)\left(D G_{2}\right)$. Therefore we can let

$$
\gamma(S, L)(q)=B_{1}(q)^{K_{S}^{2}} B_{2}(q)^{L K_{S}} B_{3}(q)^{\chi(L)} B_{4}(q)^{\chi\left(\mathcal{O}_{S}\right)} .
$$

For all generic K3 surfaces $S$ and primitive classes $L$,

$$
\gamma(S, L)(q)=\frac{\left(D G_{2}(\tau) / q\right)^{\chi(L)}}{\Delta(\tau) D^{2} G_{2}(\tau) / q^{2}} .
$$

These pairs can achieve infinitely many distinct $\chi(L)$, and thus

$$
B_{3}(q)=D G_{2}(\tau) / q \text { and } B_{4}(q)=\left(\Delta(\tau) D^{2} G_{2}(\tau) / q^{2}\right)^{1 / 2}
$$

and the result follows.

REMARK. Although in [BL] they found the formula for generating function of primitive classes on K3 surfaces with Picard number one, it is not trivial to see why the coefficients equals our universal polynomials. For each $r$, we have to show that there exist infinitely many K3 surfaces with Picard number one and primitive classes $L$ such that $L$ is $(5 r-1)$-very ample and also have distinct intersection numbers $L^{2}$. It can be proved by using Lemma 5.3 in [KP2], which shows that on a K3 surface with Picard number 
one, a primitive class $L$ is $(5 r-1)$-very ample if $L^{2}>20 r$. Therefore Bryan and Leung's generating function is exactly $\phi(S, L)(x)$.

There is a reformulation of Theorem 1.3 given by Göttsche ([Gö], remark 2.6), which is the original version of Bryan and Leung's formula.

Corollary 4.4. For all $l, m, r$, define

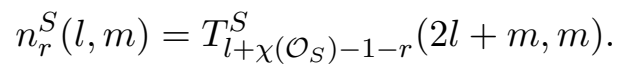

Then

$$
\sum_{l \in \mathbb{Z}} n_{r}^{S}(l, m) q^{l}=B_{1}(q)^{K_{S}^{2}} B_{2}(q)^{m}\left(D G_{2}(\tau)\right)^{r} \frac{D^{2} G_{2}(\tau)}{\left(\Delta(\tau) D^{2} G_{2}(\tau) / q^{2}\right)^{\chi\left(\mathcal{O}_{S}\right) / 2}} .
$$

If $L$ is sufficiently ample with respect to $\delta=\chi(L)-1-r$, then $n_{r}^{S}\left(\left(L^{2}-\right.\right.$ $\left.\left.L K_{S}\right) / 2, L K_{S}\right)$ counts the $\delta$-nodal curves in a general $r$-codimensional sublinear system of $|L|$. This reformulation provides another (possibly better) way to find the closed form of $B_{1}(q)$ and $B_{2}(q)$.

\section{References}

[BL] J. Bryan and C. Leung, The enumerative geometry of K3 surfaces and modular forms, J. Amer. Math. Soc. 13 (2000), 371-410.

[Bl] F. Block, Relative Node polynomials for plane curves, preprint, arXiv:1009.5063.

[Ch] Y. Choi, Severi Degrees in Cogenus 4, preprint, arXiv:alg-geom/9601013.

$[\mathrm{CH}]$ L. Caporaso and J. Harris, Counting plane curves of any genus, Invent. Math. 131 No. 2 (1998), 345-292.

[EGL] G.Ellingsrud, L. Göttsche and M. Lehn, On the cobordism class of the Hilbert scheme of a surface, Jour. Alg. Geom. 10 (2000), 81-100.

[FM] S. Fomin and G. Mikhalkin, Labeled floor diagrams for plane curves, J. Europ. Math. Soc. 12 (2010), 1453-1496.

[FI] P. Di Francesco and C. Itzykson, Quantum intersection rings in The Moduli Space of Curves, Birkhäuser, (1995), 81-148.

[Fu] W. Fulton, Intersection Theory, A series of Modern Surveys in Mathematics, Springer-Verlag, (1984).

[Gö] L. Göttsche, A Conjectural Generating Function for Numbers of Curves on Surfaces, Communications in Mathematical Physics. 196 (1998), 523-533.

[Ha] R. Hartshorne, Algebraic Geometry, Graduate Text in Mathematics, SpringerVerlag 52.

[HP] J. Harris and R. Pandharipande, Severi degrees in Cogenus 3, preprint, arXiv:alggeom/9504003.

[KM] M. Kontsevich and Y. Manin, Gromov-Witten classes, quantum cohomology, and enumerative geometry, Comm. Math. Phys. 164 (1994), 525-562.

[KP1] S. Kleiman and R. Piene, Enumerating singular curves on surfaces, Cont. Math. 241 (1999), 209-238.

[KP2] S. Kleiman and R. Piene, Node polynomials for families: methods and applications, Math. Nachr. 271 (2004), 69-90.

[KST] M. Kool, V. Shende and R. Thomas, A short proof of the Göttsche conjecture, Geom. Topol. 15, No. 1 (2011), 397-406.

[KZ] M. Kaneko and D. Zagier, A generalized Jacobi theta function and quasimodular forms in The moduli space of curves, eds. R. Dijkgraaf, C. Faber, G. van der Geer, Boston: Birkhäuser, (1995), 165-172. 
[Li] J. Li, Relative Gromov-Witten invariants and a degeneration formula of GromovWitten invariants, J.Diff. Geom, 60 (2002) 199-293.

[Liu1] A. Liu, Family blowup formula, admissible graphs and the enumeration of singular curves, I, J. Differential Geometry 56 (2000), 381-379, MR1868322, Zbl 1036.14014 .

[Liu2] A. Liu, The algebraic proof of the universality theorem, preprint, arXiv:math.AG/0402045.

[LM] M. Levine and F. Morel, Algebraic cobordism, Springer Monographs in Mathematics Springer, Berlin (2007).

[LP] M. Levine and R. Pandharipande, Algebraic cobordism revisited, Invent. Math. 276 (2009), No. 1, 63-130.

[LeeP] Y.-P. Lee and R. Pandharipande, Algebraic cobordism of bundles on varieties, preprint, arXiv:1002.1500, to appear in JEMS.

[LW] J. Li and B. Wu, Good degeneration of Quot-schemes and coherent systems, preprint, arXiv:1110.0390.

[Ran1] Z. Ran, The degree of a Severi variety, Bull. AMS. (New Series), 17 (1987), No. 1, 125-128.

[Ran2] Z. Ran, Enumerative Geometry of Singular Plane Curves, Invent. Math. 97 No. 3, (1989) 447-465.

[Tz] Y. Tzeng, A proof to the Göttsche-Yau-Zaslow formula, preprint arXiv:1009.5371, to appear in Journal of Diff. Geometry.

[Va] I. Vainsencher, Enumeration of n-fold tangent hyperplanes to a surface, Journal of Algebraic Geometry 4 (1995), 503-526.

[Vakil] R. Vakil, Counting curves on rational surfaces, Manuscripta Mathematica, 102 No. 1, (2000), 53-84.

[YZ] S. T. Yau and E. Zaslow, BPS states, string duality, and nodal curves on K3, Nuclear Physics B. 471 No. 3, (1996), 503-512.

Department of Mathematics, Harvard University, Cambridge, MA 02138, USA

E-mail address: ytzeng@math.harvard.edu 
